\title{
Metabolism of $\omega$-Conotoxin-sensitive Voltage-operated Calcium Channels in Human Neuroblastoma Cells: Modulation by Cell Differentiation and Anti-Channel Antibodies
}

\author{
M. Passafaro, ${ }^{2}$ F. Clementi, ${ }^{1}$ and E. Sher' \\ ${ }^{1}$ CNR Center of Cytopharmacology, Department of Medical Pharmacology, University of Milan, 20129 Milan, Italy and \\ 2Department of Biology, Faculty of Science, University of Rome "Tor Vergata," 00173 Rome, Italy
}

The turnover of voltage-operated calcium channels was studied in two different human neuroblastoma cell lines (IMR32 and SH-SY5Y) using $\omega$-conotoxin. The ${ }^{125} / \omega$-conotoxin bound to surface channels was internalized and degraded by the cells in a time- and temperature-dependent manner. The radioactive degradation products released in the medium were all trichloroacetic acid soluble and no longer recognized by anti- $\omega$-conotoxin antibodies. Altering the pH of intracellular organelles with chloroquine and inhibiting lysosomal proteases with leupeptin reduced ${ }^{125} \mid-\omega$-conotoxin degradation but had no effect on its internalization. Postlabeling measurements showed that the rates of ${ }^{125} \mid-\omega$-conotoxin internalization and degradation were equal to the rate of channel removal from the cell surface after protein synthesis inhibition. The rate of removal of $\omega$-conotoxin binding sites was parallel to the rate of loss of functional channels, as measured by means of the fura-2 technique.

Drug-induced differentiation of human neuroblastoma cells slowed down channel internalization and degradation rates, leading to the known increased expression of plasma membrane calcium channels in differentiated cells. On the other hand, both human (from Lambert-Eaton myasthenic patients) and murine (from immunized mice) anti-channel antibodies increased the rates of channel internalization and degradation, leading to channel downregulation.

The activity of presynaptic calcium channels is already known to be acutely modulated by a number of different agents (e.g., hormones and neurotransmitters); our studies suggest that a different form of channel modulation (changes in the number of channels due to interference with channel turnover) may be active over a longer time scale in neurons. This form of modulation could be important in both physiological and pathological states.

A crucial ion channel on the plasma membrane of nerve terminals is the voltage-operated calcium channel (VOCC), which, upon depolarization, opens its pore and allows calcium ions to

\footnotetext{
Received Oct. 9, 1991; revised Mar. 2, 1992; accepted Mar. 27, 1992.

This work was supported by Italian National Research Council Special Project "Mechanisms of Neurotransmitter Release" to E.S. and Telethon Program 1991: "Combatti la Distrofia Musculare" to F.C. We thank Prof. F. Di Virgilio and Dr. C. Gotti for their helpful suggestions; Prof. G. Fumagalli and Prof. N. Borgese for critically reading the manuscript; Dr. E. Biancardi, who participated in the preliminary experiments; and Mr. P. Tinelli for his technical help.

Correspondence should be addressed to Dr. E. Sher, CNR Center of Cytopharmacology, via Vanvitelli 32, 20129 Milano, Italy.

Copyright (C) 1992 Society for Neuroscience $0270-6474 / 92 / 123372-08 \$ 05.00 / 0$
}

enter the nerve terminal along their electrochemical gradient, thus triggering neurotransmitter release by exocytosis.

Although a lot of information is available on the functional and pharmacological properties of neuronal VOCCs (for recent reviews, see Bean, 1989; Hess, 1990; Sher et al., 1991), few reports have been published on the cellular mechanisms controlling VOCC expression, subcellular localization, and turnover in neuronal cells.

VOCC expression has been shown to change during in vitro neuronal differentiation (Streit and Lux, 1989; Carbone et al., 1990; Usowicz et al., 1990), and a subcellular compartmentalization of specific VOCC subtypes in different domains of the plasma membrane has recently been demonstrated (Ahlidanian et al., 1990; Robitaille et al., 1990; Westenbroek et al., 1990; Cohen et al., 1991; Torri Tarelli et al., 1991), but the cellular mechanisms underlying these processes are still poorly characterized. This is mainly due to the previous lack of specific ligands and suitable in vitro cellular systems for studying VOCC metabolism.

We have now overcome this problem by using the calcium channel antagonist $\omega$-conotoxin ( $\omega$-ctx) in human neuroblastoma cell lines expressing a sufficient number of VOCCs.

Neuroblastoma cells have already been shown to be a useful cellular model for studying the pharmacology and physiology of human neuronal VOCCs (Sher et al., 1988; Carbone et al., 1990). Furthermore, these cells have the interesting properties of developing a secretory apparatus after exposure to differentiating agents (Sher et al., 1989a), and of forming cholinergic synapses when cocultured with muscle cells (Thompson et al., 1982).

$\omega$-Ctx is a peptide neurotoxin present in the venom of fishhunting marine snails of the Conus genus, which has been recently shown to block selectively one subtype of the high-voltage-activated calcium channels present in neurons and other secretory cells (Olivera et al., 1990; Sher and Clementi, 1991). We (Torri Tarelli et al., 1991) and others (Robitaille et al., 1990; Cohen et al., 1991) have shown that $\omega$-ctx-sensitive VOCCs (also referred to as $\omega$-type VOCCs) are clustered in the presynaptic active zones; this, together with a number of other functional data (reviewed in Miller, 1987; Sher and Clementi, 1991), suggests that this VOCC subtype plays a critical rolc in controlling neurotransmitter release.

This article describes the general properties of $\omega$-type VOCC turnover and demonstrates that it can be modulated during cellular differentiation or by pathological events.

Although information has long been available on the metab- 
olism of other membrane ion channels, such as the nicotinic ACh receptor (Fambrough, 1979) and, to a lesser extent, the voltage-operated sodium channel (Sherman and Catterall, 1984; Bar-Sagi and Prives, 1985; Dargent and Couraud, 1990), this is the first detailed study of the metabolism of a neuronal VOCC subtype.

\section{Materials and Methods}

\section{Cell culture and differentiation}

The IMR32 cell line (ATCC CCL127) was obtained from the American Type Culture Collection (Rockville, MD), and grown and differentiated as previously described (Sher et al., 1989a).

The SH-SY5Y cell line (Pahlman et al., 1984) was kindly provided by Dr. M. Toselli (University of Pavia). These cells were grown in RPMI 1640 medium supplemented with $10 \%$ fetal calf serum, $100 \mathrm{IU} / \mathrm{ml}$ penicillin, and $100 \mu \mathrm{g} / \mathrm{ml}$ streptomycin. Their differentiation was achieved by adding a final concentration of $10 \mu \mathrm{M}$ retinoic acid freshly dissolved in ethanol directly in the dishes.

The cells were kept at $37^{\circ} \mathrm{C}$ in a humidified incubator at a $5 \% \mathrm{CO}_{2}$ concentration in air. For immunoprecipitation experiments, the cells were grown to confluency in Falcon 100-mm-diameter plastic Petri dishes; for turnover experiments, the cells were plated at a concentration of $3 \times 10^{5}$ in $35-\mathrm{mm}$-diameter dishes and used after the indicated times.

\section{${ }^{125} I-\omega-c t x$ binding assay}

In order to he able to perform experiments on intact adherent cells, a modification of a previously described binding assay protocol (Sher et al., 1988) was used. Cells grown in 35-mm-diameter dishes were washed twice with Dulbecco's modified phosphate-buffered saline containing $0.1 \%$ bovine serum albumin (DPBS-BSA) $1 \mathrm{ml} / \mathrm{dish} .{ }^{125} \mathrm{I}-\omega$-ctx was added to either the DPBS-BSA or the culture medium at the indicated concentrations (usually 25, 50, or $100 \mathrm{pm}$ ) and left on the cells for $2 \mathrm{hr}$ at room temperature. After five washes with DPBS-BSA, $1 \mathrm{ml} / \mathrm{dish}$, to remove unbound toxin, the cells were extracted in $1 \mathrm{ml}$ of $1 N \mathrm{NaOH}$ and bound radioactivity was determined by means of a Beckman $\gamma 4000$ gamma-counter. Each binding point was evaluated on triplicate dishes. Nonspecific ${ }^{125} \mathrm{I}-\omega$-ctx binding was evaluated for every group by incubating three parallel dishes in the presence of an excess $(200 \mathrm{~nm})$ of unlabeled toxin. Under these conditions, nonspecific ${ }^{125} \mathrm{I}-\omega$-ctx binding was $\approx 15 \%$ of total hinding.

\section{${ }^{125} I-\omega-c t x-V O C C$ complex degradation}

In order to study the degradation of prebound ${ }^{125}$ I- $\omega$-ctx, different groups of dishes were washed and labeled as described above. In each group, total and nonspecific binding were evaluated in triplicate. One group was extracted as described above in order to evaluate specific ${ }^{125} I-\omega$-ctx binding at time 0 . The other groups were returned to the incubator at $37^{\circ} \mathrm{C}$ (or at the temperatures indicated in the figures) in the prescnce of control culture medium. In some experiments, the culture medium was supplemented with drugs (see figure captions). At the indicated times, a single group of dishes was recovered; the medium was collected, centrifuged, and counted to determine released radioactivity; and the cells were extracted to determine the amount of remaining cell-associated radioactivity.

\section{${ }^{25} I-\omega-c t x-V O C C$ complex internalization}

In order to measure ${ }^{125} I-\omega$-ctx cell internalization, we used an acid wash procedure similar to the one described in other models of receptor internalization (Vuk-Pavlovic and Kovach, 1989).

We first determined the buffer and $\mathrm{pH}$ conditions that allowed us to detach the greatest amount of surface-bound ${ }^{125} I-\omega$-ctx without altering cell viability. Two $5 \mathrm{~min}$ washes with $0.5 \mathrm{ml}$ of a $1 \%$ acetic acid buffer, pH 3 , detached $\approx 90 \%$ of surface-bound ${ }^{125}$ I- $\omega$-ctx without significantly altering cell viability (trypan blue exclusion indicated that $<5 \%$ of the cells were killed). Higher $\mathrm{pH}$ values did not detach bound ${ }^{125} \mathrm{I}-\omega-\mathrm{ctx}$, and longer incubations increased cell mortality (not shown).

To determine ${ }^{125} I-\omega$-ctx internalization rates, the cells were labeled as described above and returned to the incubator in the presence of control culture medium or one of the different agents to be tested. At the indicated times, a single group of cells was recovered and the medium collected and counted to determine ${ }^{125} I-\omega$-ctx degradation. The cells were then washed with the acid buffer, which was collected, centrifuged, and counted in order to determine the amount of "acid-releasable" ${ }^{125}$ I$\omega$-ctx (i.e., still on the cell surface). The cells were then extracted as described above in order to determine the amount of "acid-resistant" ${ }^{125}$ I- $\omega$-ctx (i.e., internalized).

\section{Characterization of released radioactivity}

TCA experiments. $\Lambda$ protocol similar to that described by Paul et al. (1989) was used to characterize released radioactivity and to test whether this really represented the degradation of ${ }^{125} \mathrm{I}-\omega$-ctx (a 27 amino acid peptide) into smaller components.

In a total volume of $300 \mu \mathrm{l}$ of culture medium, we added either native ${ }^{125} \mathrm{I}-\omega \cdot \operatorname{ctx}(50 \mathrm{pM}, \approx 3000 \mathrm{cpm} / \mathrm{ml})$ or $\approx 3000 \mathrm{cpm}$ of the radioactivity contained in the medium of the cells incubated for $24 \mathrm{hr}$ at $37^{\circ} \mathrm{C}$ after the labeling procedure (putative degraded ${ }^{125} \mathrm{I}-\omega$-ctx). Parallel controls were performed by adding identical amounts of radioactivity corresponding to native ${ }^{125} \mathrm{I}-\omega$-ctx incubated $24 \mathrm{hr}$ at $37^{\circ} \mathrm{C}$ in the absence of cells, but either with control culture medium, or with "conditioned medium" (i.e., medium collected from routinely cultured IMR 32 cells, in which putative released proteolytic enzymes might be present). A 1 $\mathrm{mg} / \mathrm{ml}$ concentration of BSA was added to each tube as carrier protein, followed by a $10 \%$ final concentration of cold trichloroacetic acid (TC $\Lambda$ ). After overnight incubation at $4^{\circ} \mathrm{C}$, the tubes were centrifuged $(10 \mathrm{~min}$ at $10,000 \mathrm{rpm}$ ), the pellets counted in a gamma-counter, and the percentage of TCA-insoluble radioactivity determined.

Immunoprecipitation with anti- $\omega$-ctx antibodies. In the presence of control rabbit serum to ensure a constant IgG level in each tube, increasing concentrations of a rabbit anti- $\omega$-ctx antiserum were added to a fixed amount of native ${ }^{125} \mathrm{I}-\omega$-ctx $(50 \mathrm{pM}, \approx 3000 \mathrm{cpm} / \mathrm{ml})$, or to a similar amount of radioactivity released in the medium by prelabeled cells, or related to the controls described above for TCA experiments. After overnight incubation at $4^{\circ} \mathrm{C}, 35 \mu$ of goat anti-rabbit IgG antibodies were added to each tube. After an additional $2 \mathrm{hr}$ at room temperature, the tubes were centrifuged, and the pellets washed once with DPBS-BSA and then counted.

\section{Antisera}

The production and properties of the anti- $\omega$-ctx antiserum were described in a previous article (Torri Tarelli et al., 1991). Lambert-Eaton myasthenic syndrome (LEMS) sera were obtained from patients with clinically diagnosed disease.

Mouse anti- $\omega$-type VOCC antibodies were obtained by intraperitoneally immunizing C 57 black mice with intact neuroblastoma cells $(\approx 0.5$ $\mathrm{ml} /$ injection, $\approx 5 \times 10^{\circ}$ cells $/ \mathrm{ml}$ ) or unrelated cells as control. After four injections ( 1 /week), the serum was collected and the anti- $\omega$-type VOCC antibody titer determined as described below.

\section{$\omega$-Type VOCC immunoprecipitation}

The titer of anti- $\omega$-type VOCC antibodies in both LEMS and mouse sera was determined by a radioimmunometric procedure we have recently developed (Sher et al., 1989b).

Briefty, a large number of neuroblastoma cells $\left(\approx 40 \times 10^{6} / \mathrm{ml}\right)$ was homogenized in a buffer consisting of $0.3 \mathrm{~m}$ sucrose, $5 \mathrm{~mm}$ HEPES/

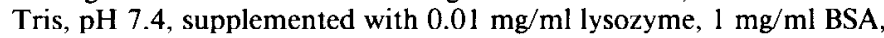
$0.5 \mathrm{~mm}$ phenylmethylsulfonyl fluoride, $2 \mu \mathrm{M}$ leupeptin, and $1 \mu \mathrm{M}$ pepstatin A. A saturating concentration ( $100 \mathrm{pm}$ ) of ${ }^{125} \mathrm{I}-\omega$-ctx was added for $2 \mathrm{hr}$ at room temperature. ${ }^{125} \mathrm{I}$ - $\omega$-ctx-VOCC complexes were then solubilized by the addition of Triton X-100 ( $1 \%$ final concentration). After $3 \mathrm{hr}$ at $4^{\circ} \mathrm{C}$, the sample was centrifuged for 30 min at $15,000 \mathrm{rpm}$ in a Sorvall RB centrifuge and the supernatant used as the source of antigen. Increasing concentrations of either LEMS or mouse serum were added to a constant volume of supernatant in the presence of control human or mouse serum as a carrier. After overnight incubation at $4^{\circ} \mathrm{C}$, $50 \mu$ l of either rabbit anti-human IgG or goat anti-mouse IgG solutions were added to each tube. After 2 additional hours at room temperature, the samples were centrifuged, the pellets washed, and the amount of precipitated radioactivity counted in a gamma-counter. The antibody titer was determined from the linear part of the precipitation curve and expressed as picomoles of ${ }^{125} \mathrm{I}-\omega$-ctx binding sites precipitated/liter of serum.

\section{$\left[\mathrm{Ca}^{2+}\right]_{\mathrm{i}}$ measurements with fura-2}

A protocol similar to the one previously described (Sher et al., 1988) was used for studying the depolarization-induced increase in $\left[\mathrm{Ca}^{2+}\right]_{i}$ and its reduction after protein synthesis blockade with cycloheximide. Brief- 


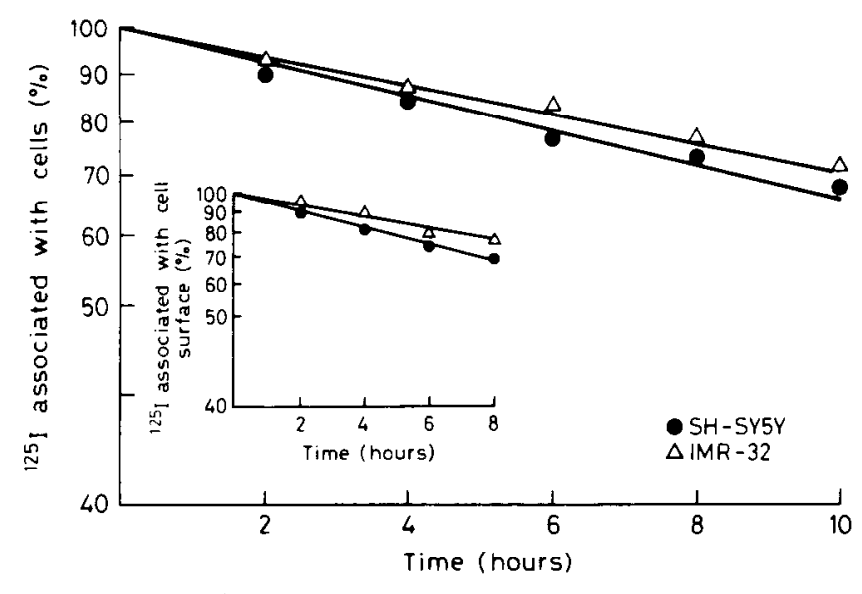

Figure 1. Degradation and internalization of ${ }^{125} I-\omega$-ctx by prelabeled cells. The figure shows the fraction of cell-associated radioactivity remaining at different times after labeling surface VOCCs with ${ }^{125} \mathbf{I}-\omega$-ctx (25 pM). Each time point is the average of five $35 \mathrm{~mm}$ culture dishes and represents the binding and degradation of only specifically bound toxin from both IMR32 $(\triangle)$ and SH-SY $5 Y(\bullet)$ cells. Specific ${ }^{125} I-\omega$-ctx binding at time 0 was $1660 \mathrm{cpm} / \mathrm{dish}$ for IMR 32 and $2525 \mathrm{cpm} / \mathrm{dish}$ for SH-SY5Y cells. In these particular experiments, the degradation half-lives for IMR32 and SH-SY5Y cells were, respectively, 19.6 and $15.9 \mathrm{hr}$. Inset, The fraction of radioactivity associated with the cell surface (acid releasable) is evaluated at different times after labeling surface VOCCs with ${ }^{125} \mathrm{I}-\omega$-ctx ( $25 \mathrm{pM}$ ). In these particular experiments, the internalization half-lives for IMR 32 and SH-SY5Y cells were, respectively, 18.2 and $15.2 \mathrm{hr}$.

ly, at different times after addition of cycloheximide, IMR 32 cells were gently detached from the dish, washed once in Krebs-Ringer-HEPES solution (containing $125 \mathrm{~mm} \mathrm{NaCl}, 5 \mathrm{~mm} \mathrm{KCl}, 12 \mathrm{mM} \mathrm{MgSO}_{4}, 1.2 \mathrm{~mm}$ $\mathrm{KH}_{2} \mathrm{PO}_{4}, 2 \mathrm{~mm} \mathrm{CaCl}, 6 \mathrm{~mm}$ glucose, and $25 \mathrm{~mm}$ HEPES-NaOH $(\mathrm{pH}$ $7.4)$, and then resuspended $\left(10^{7} \mathrm{cells} / \mathrm{ml}\right)$ in the same solution. Loading of the cells with fura- 2 was achieved by incubating the cell suspension with $2.5 \mu \mathrm{M}$ fura- 2 acetoxymethyl ester for $30 \mathrm{~min}$ at $37^{\circ} \mathrm{C}$. Fluorescent measurements (excitation and emission, respectively, at 345 and 490 $\mathrm{nm}$ ) were made in a spectrofluorometer using a thermostatically controlled cell holder equipped for continuous stirring. Calibration of the fluorescent signal in terms of $\left[\mathrm{Ca}^{2+}\right]_{i}$ was performed as previously described (Di Virgilio et al., 1987).

\section{Reagents}

Minimum essential medium, RPMI 1640, fetal calf serum, antibiotics, and glutamine were obtained from Flow Labs (Ayrshire, UK). Plastic Falcon petri dishes were purchased from Becton Dickinson (Plymouth, UK). Dibutyryl-5-cyclic monophosphate, 5-bromodeoxyuridine, retinoic acid, BSA, anti-rabbit IgG, anti-human IgG, anti-mouse IgG, leupeptin, pepstatin A, carbachol, and cycloheximide were purchased from Sigma Chemical Co. (St. Louis, MO). Synthetic $\omega$-ctx fraction GVIA was obtained from Bachem (Bubendorf, Switzerland), while ${ }^{125} I-\omega-c t x$ was from Amersham (Amersham, UK). Chloroquine was from Boehringer Mannheim (Germany), and fura-2 acetoxymethyl ester was from Molecular Probes (Eugene, OR). All other reagents were of reagent grade and were purchased from E. Merck (Darmstadt, Germany).

\section{Results}

${ }^{125} I-\omega-c t x-V O C C$ complex degradation

In a previous study, we showed that the specific and saturable binding of ${ }^{125}[-\omega$-ctX to human neuroblastoma cells is essentially irreversible (Sher et al., 1988). Furthermore, parallel changes in the number of ${ }^{125} I-\omega-c t x$ binding sites and in the amount of $\omega$-ctx-sensitive calcium currents have been described in different cells (Carbone et al., 1990; E. Sher, E. Carbone, E. Biancardi, and F. Clementi, unpublished observations), confirming the validity of using this toxin as a marker for calcium channels.

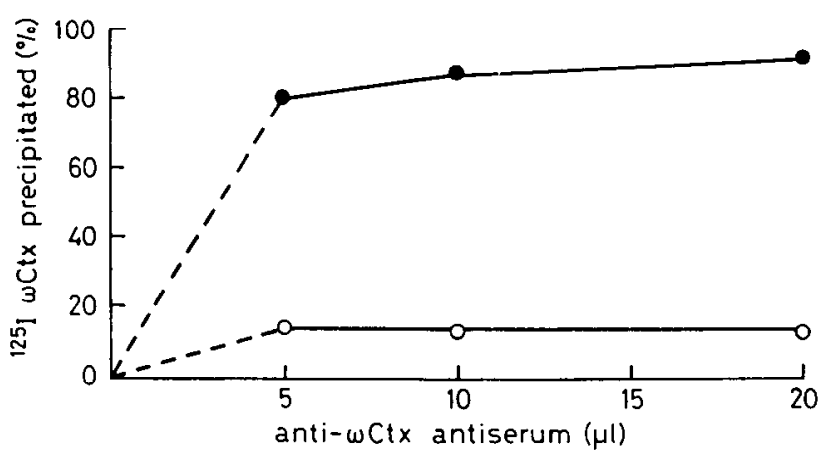

Figure 2. Immunoprecipitation of ${ }^{125} \mathrm{I}-\omega$-ctx and its degradation products. Increasing concentrations of anti-toxin antibodies were added either to a fixed amount of native ${ }^{125} \mathrm{I}-\omega$-ctx $(\Theta)$ or to the samc amount of radioactivity released in the medium by prelabeled cells (O). Antitoxin antibodies precipitated most of the native toxin, but only a small fraction of released radioactivity. Each point was performed in quadruplicate, and similar results were obtained in three independent experiments.

Taking advantage of these properties, intact, living neuroblastoma cells were labeled with ${ }^{125} \mathrm{I}-\omega$-ctx, and after extensive washing, the fate of the irreversibly bound toxin at $37^{\circ} \mathrm{C}$ was followed, as an indirect estimate of $\omega$-type VOCC turnover. Similar "prelabeling" protocols have already been fruitfully used for the study of nicotinic ACh receptor turnover in both muscle (Devreotes and Fambrough, 1975) and chromaffin cells (Higgins and Berg, 1988).

VOCC turnover was evaluated by measuring both the radioactivity released in the medium and that remaining associated with the cells in groups of dishes recovered at different times after labeling.

Specifically bound radioactivity (total minus nonspecific) was released in the medium with a monoexponential kinetic. Large quantities of nonspecific radioactivity were released during the first $2 \mathrm{hr}$ at $37^{\circ} \mathrm{C}$ and in negligible amounts thereafter, thus probably reflecting a washout phenomenon of the ${ }^{125} \mathrm{I}-\omega$-ctx nonspecifically bound to the dishes. At $37^{\circ} \mathrm{C}$, the half-life values of the radioactivity released from the cells were $16.5 \pm 0.8 \mathrm{hr}$ (mean $\pm \mathrm{SEM} ; n=18$ ) and $16 \pm 1.3 \mathrm{hr}$ (mean $\pm \mathrm{SEM} ; n=4$ ) for undifferentiated SH-SY5Y and IMR32 cells, respectively (Fig. 1).

The rate of radioactivity release was not influenced by either the ${ }^{125} I-\omega$-ctx concentration used for labeling surface VOCCs (between 10 and $50 \mathrm{pm}$ ), or the duration of the labeling step (between 15 and $120 \mathrm{~min}$ ) (not shown). Furthermore, the presence or absence of fetal calf serum in the medium did not acutely affect ${ }^{125} I-\omega$-ctx degradation rate.

The nature of the released radioactive material was investigated by means of TCA precipitation experiments and with the aid of anti- $\omega$-ctx antibodies. TCA (10\%) precipitated only a small fraction of the radioactivity released in the medium by prelabeled cells after $24 \mathrm{hr}$ at $37^{\circ} \mathrm{C}(\approx 10 \%$ of the precipitable native ${ }^{125}[-\omega-c t x)$. This demonstrates that most of the released radioactivity is associated with small, TCA-soluble degradation fragments of ${ }^{125} I-\omega$-ctx. Native ${ }^{125} I-\omega$-ctx, incubated for $24 \mathrm{hr}$ at $37^{\circ} \mathrm{C}$ in the absence of cells but in the presence of either fresh or "conditioned" medium, was not degraded and could still be precipitated to the same degree as native ${ }^{125} \mathrm{I}-\omega$-ctx (not shown). Anti- $\omega$-ctx antibodies were able to precipitate more than $90 \%$ of the radioactivity due to native ${ }^{125} \mathrm{I}-\omega$-ctx , but precipitated less than $10 \%$ of the same amount of radioactivity collected after 


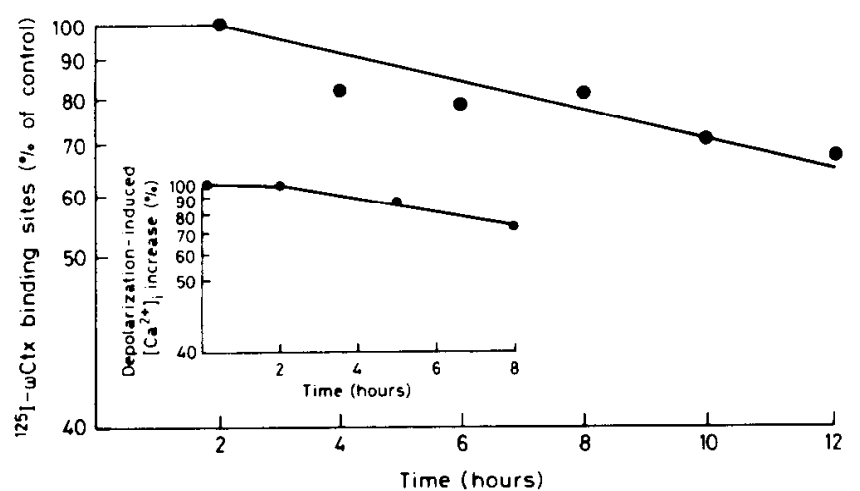

Figure 3. Loss of surface ${ }^{125} \mathrm{I}-\omega$-ctx binding sites and functional $\mathrm{Ca}^{2+}$ channels in cycloheximide-treated cells. The results represent the fraction of surface ${ }^{125} I-\omega$-ctx binding sites still available after blocking protein synthesis with cycloheximide $(100 \mu \mathrm{g} / \mathrm{ml})$ at time 0 . Each time point represents the average of five $35 \mathrm{~mm}$ culture dishes, and only specific binding is plotted. ${ }^{125} \mathrm{I}-\omega$-ctx-specific binding at time 0 was 1254 $\mathrm{cpm} /$ dish in this particular experiment performed on IMR32 cells. In this representative experiment, the half-life for loss of binding sites was $18.0 \mathrm{hr}$. Similar results were obtained in eight independent experiments. Inset, The fraction of depolarization-induced $\left[\mathrm{Ca}^{3+}\right]_{i}$ increase still available after blocking protein synthesis with cycloheximide as evaluated at different times. The half-life for loss of functional $\mathrm{Ca}^{2+}$ channels was $15.6 \mathrm{hr}$. Each point represents the average of five independent determinations.

$24 \mathrm{hr}$ at $37^{\circ} \mathrm{C}$ from the medium of prelabeled cells (Fig. 2). More than $80 \%$ of the ${ }^{125} \mathrm{I}-\omega$-ctx incubated for $24 \mathrm{hr}$ at $37^{\circ} \mathrm{C}$ with either fresh culture or "conditioned" medium was still precipitable by the antibodies (not shown).

This result indicates that most of the radioactivity released in the medium by prelabeled cells at $37^{\circ} \mathrm{C}$ is related to the proteolysis of ${ }^{125} \mathrm{I}-\omega$-ctx, and not simply to its dissociation from the cells.

\section{${ }^{225} I-\omega-c t x-V O C C$ complex internalization}

To determine whether ${ }^{125} \mathrm{I}-\omega$-ctx degradation was coupled to VOCC internalization, two different strategies were used: acid wash and protein synthesis inhibition.

\section{Table 1. Effect of cycloheximide treatment on depolarization and carbachol-induced increase in $\left[\mathrm{Ca}^{2+}\right]_{i}$}

\begin{tabular}{lclll}
$\begin{array}{l}\text { Time in } \\
\text { cyclo- } \\
\text { heximide } \\
\text { (hr) }\end{array}$ & Basal & $\begin{array}{l}\mathrm{KCl} \\
(60 \mathrm{~mm})\end{array}$ & $\begin{array}{l}\text { \% Incresae } \\
\text { over basal }\end{array}$ & $\begin{array}{l}\text { \% Re- } \\
\text { mainin }\end{array}$ \\
\hline- & $82 \pm 12.5$ & $173 \pm 37$ & $193 \pm 7.5$ & 100 \\
2 & $77 \pm 2.6$ & $197 \pm 9$ & $189 \pm 4.8$ & 98.5 \\
5 & $80 \pm 9.9$ & $143 \pm 16.3$ & $172 \pm 6.9$ & 88.2 \\
8 & $102 \pm 11$ & $140 \pm 14.9$ & $144 \pm 2.5$ & 73.8 \\
CCh & & & & \\
$(100 \mu \mathrm{M})$ & & & & \\
- & $84 \pm 13.8$ & $190 \pm 36$ & $221 \pm 9.4$ & 100 \\
2 & $70 \pm 5.1$ & $170 \pm 18.1$ & $240 \pm 14.8$ & 108 \\
5 & $89.1 \pm 18.7$ & $237 \pm 83.2$ & $244 \pm 27.3$ & 110 \\
8 & $101 \pm 6.9$ & $236 \pm 30.1$ & $254 \pm 10.2$ & 114
\end{tabular}

Fura- 2 measurements of $\left[\mathrm{Ca}^{2+}\right]$ and its modulation by the depolarization-induced opening of VOCCs $(60 \mathrm{mM} \mathrm{KCl})$ or by muscarinic receptor activation $(100 \mu \mathrm{M}$ carbachol) were studicd as described in Materials and Methods. Both basal and stimulated values are expressed as nanomolar calcium concentrations. Each value represents the average \pm SE obtained from five independent determinations.

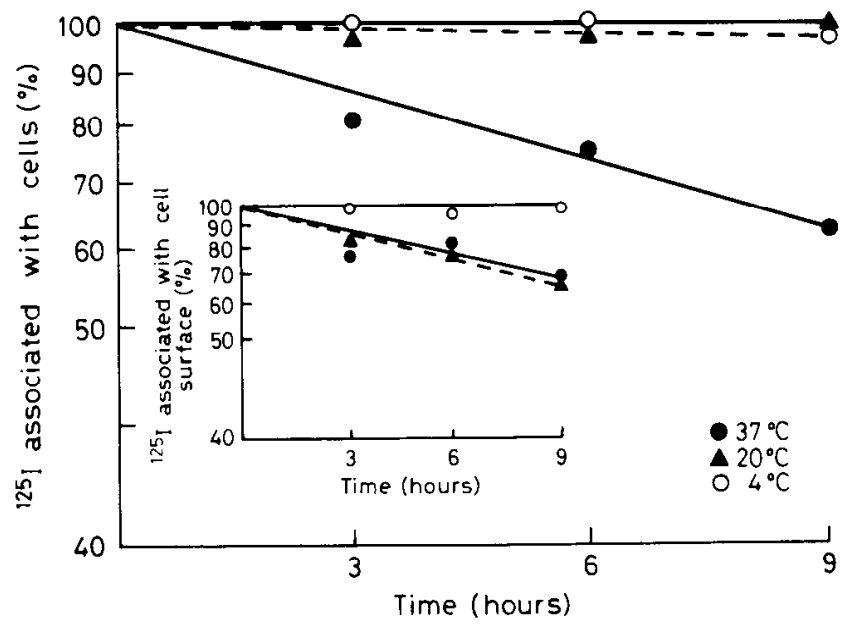

Figure 4. Effects of temperature on ${ }^{125} \mathrm{I}-\omega$-ctx degradation and internalization. Degradation of ${ }^{125} \mid-\omega$-ctx by prelabeled cells is prevented by incubating SII-SY $5 Y$ neuroblastoma cells at $20^{\circ} \mathrm{C}(\Delta)$ or $4^{\circ} \mathrm{C}(\mathrm{O})$. Inset, Internalization of ${ }^{125} \mathrm{I}-\omega$-ctx is blocked only at $4^{\circ} \mathrm{C}(\mathrm{O})$ but not at $20^{\circ} \mathrm{C}$ (A). Control cells at $37^{\circ} \mathrm{C}$. These results are representative of five experiments giving similar results.

The neuroblastoma cells were prelabeled with ${ }^{125} \mathrm{I}-\omega-\mathrm{ctx}$ and returned to $37^{\circ} \mathrm{C}$ as described above for the degradation experiments; at the indicated times, the internalized (acid-resistant) and surface-bound (acid-releasable) specific radioactivity was measured.

The half-lives for ${ }^{25} \mathrm{I}-\omega$-ctx internalization (in control medium and at $37^{\circ} \mathrm{C}$ ) werc $13.5 \pm 0.6 \mathrm{hr}$ (mean $\pm \mathrm{SEM} ; n=11$ ) for undifferentiated SH-SY $5 Y$ cells and $15.3 \pm 2.4 \mathrm{hr}$ (mean \pm SEM; $n=4$ ) for undifferentiated IMR32 cells (Fig. 1, inset). These are similar to the half-lives obtained measuring radioactivity release into the medium, which suggests that, at least under control conditions, clearance of ${ }^{125} \mathrm{I}-\omega$-ctx from the cell surface and radioactivity release may be coupled processes.

It was also important to demonstrate that $\omega$-type VOCC degradation occurs in parallel to that of the bound ${ }^{125} \mathrm{I}-\omega$-ctx. Consequently, we studied the time course of both the removal of toxin binding sites from the cell surface and the loss of the depolarization-induced increase in $\left[\mathrm{Ca}^{2+}\right]_{i}$ after protein synthesis was blocked with cycloheximide (postlabeling protocol and fura- 2 measurements). Figure 3 shows that, after a lag of $\approx 2$ $\mathrm{hr}$, IMR32 cells incubated in the presence of $100 \mu \mathrm{g} / \mathrm{ml}$ cycloheximide lost their toxin binding sites with a half-life of 17.5 $\pm 1.8 \mathrm{hr}($ mcan $\pm \mathrm{SEM} ; n=10)$, a value similar to the rate of release of radioactivity into the medium by prelabeled cells.

The KCl-induced increase in [ $\left.\mathrm{Ca}^{2+}\right]_{i}$ in IMR32 cells was more than $90 \%$ blocked by 100 nM $\omega$-ctx but only partially affected by $1 \mu \mathrm{M}$ verapamil (not shown). Therefore, $\mathrm{KCl}$-induced $\mathrm{Ca}^{2+}$ influx under these conditions can be taken as a good measure of the functional $\omega$-type VOCC present on the plasma membrane. Figure 3 (inset) shows that after protein synthesis blockade, the number of functional channels present on the plasma membrane declines with a kinetic similar to the loss of surface toxin binding sites. Also in this case, channel loss started after a lag of $\approx 2 \mathrm{hr}$ and the measured half-life was $16.5 \mathrm{hr}$, similar to the rate of internalization measured with binding techniques. As shown in Table 1, cycloheximide treatment for up to $8 \mathrm{hr}$ did not significantly affect basal $\left[\mathrm{Ca}^{2+}\right]_{i}$, but specifically decreased the ability of $\mathrm{KCl}$ to increase $\left[\mathrm{Ca}^{2+}\right]_{i}$. On the same time 


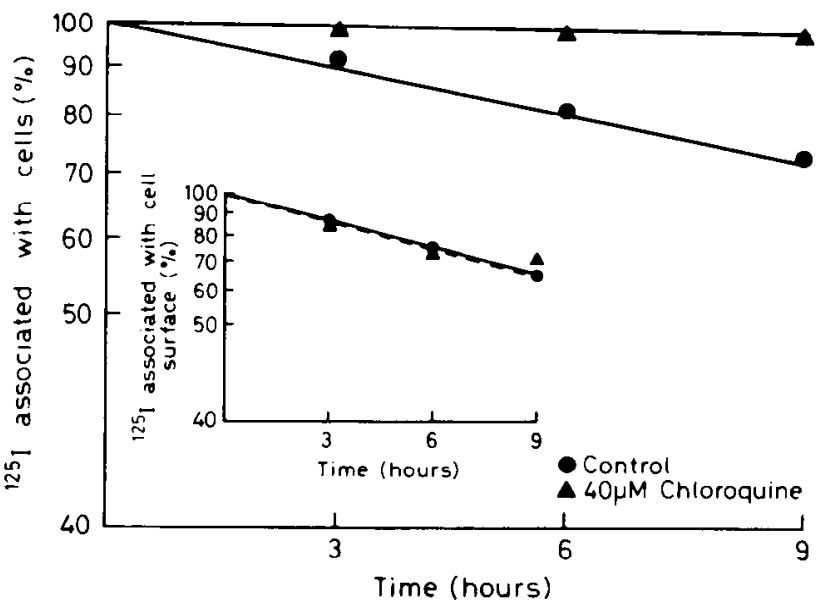

Figure 5. Effects of chloroquine on ${ }^{125} 1-\omega$-ctx degradation and internalization. At $37^{\circ} \mathrm{C}$, chloroquine $(40 \mu \mathrm{M} ; \Delta)$ prevents ${ }^{125} \mathrm{I}-\omega$-ctx degradation but not ${ }^{125} \mathrm{I}-\omega$-ctx internalization (inset) in undifferentiated $\mathrm{SH}-$ SY5Y cells. In these representative experiments, control half-lives (๑) for degradation and internalization were, respectively, 15.3 and 14.4 hr. Half-lives in the presence of chloroquine were, respectively, 41.0 and $15.5 \mathrm{hr}$. Similar results were obtained in three independent experiments.

scale, no reduction in the carbachol-induced increase in $\left[\mathrm{Ca}^{2+}\right]_{\text {, }}$ could be detected (Table 1), suggesting that VOCCs and muscarinic receptors in IMR32 cells might have different turnover rates.

The good agreement between these different values, measured using both binding and fura-2 techniques, strongly suggests (1) that the release of radioactivity into the medium is a result of ${ }^{125} \mathrm{I}-\omega$-ctx-VOCC complex degradation and therefore can be taken as an indirect measure of VOCC turnover, and (2) that $\omega$-ctx binding per se does not significantly affect VOCC turnover.

\section{Effect of temperature and lysosomotropic drugs on ${ }_{12 .} I-\omega-c t x-V O C C$ complex internalization and degradation}

The results shown above are compatible with a model in which the ${ }^{125} I-\omega$-ctx bound to the channel is internalized by endocytosis and then transported to the lysosomes, where it is hydrolized and the radioactive degradation products are released into the medium. To test this hypothesis further, ${ }^{125} \mathrm{I}-\omega$-ctx internalization and degradation rates were measured in cells exposed to low temperatures or to drugs known to affect the lysosomal degradation of proteins.

${ }^{125} \mathrm{I}-\omega$-ctx degradation was completely blocked when prelabeled cells were incubated at either $4^{\circ} \mathrm{C}$ or $20^{\circ} \mathrm{C}$ (Fig. 4), whereas ${ }^{125} \mathrm{I}-\omega$-ctx internalization was blocked only at $4^{\circ} \mathrm{C}$, and at $20^{\circ} \mathrm{C}$ the internalization rate remained similar to that of the $37^{\circ} \mathrm{C}$ control (Fig. 4, inset). Endosome-lysosome fusion is blocked at $<20^{\circ} \mathrm{C}$ (Van Deurs et al., 1989); therefore, our data suggest that the degradation of the ${ }^{125} \mathrm{I}-\omega$-ctx-VOCC complex only occurs after transport to lysosomes.

The incubation of ${ }^{125} \mathrm{I}-\omega$-ctx prelabeled cells in a medium containing chloroquine, a drug that raises the $\mathrm{pH}$ of intraccllular organelles, led to a dramatic slowing down of radioactivity release into the medium (Fig. 5). The effect of chloroquine was dose dependent (not shown). At $37^{\circ} \mathrm{C}$, in the presence of $20 \mu \mathrm{M}$ chloroquine the degradation half-life in IMR32 cells was $47 \pm$ $5.9 \mathrm{hr}$ (mean $\pm \mathrm{SEM} ; n=4$ ) (Fig. 5), significantly longer than in the control. Leupeptin, a selective inhibitor of acid hydrolases

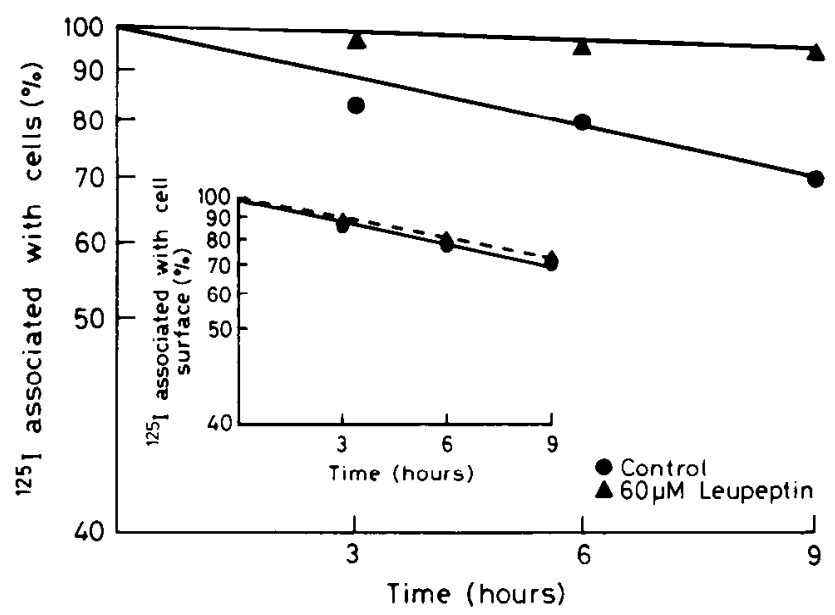

Figure 6. Effects of leupeptin on ${ }^{125} I$ - $\omega$-ctx degradation and internalization. At $37^{\circ} \mathrm{C}$, leupeptin $(60 \mu \mathbf{M} ; \mathbf{\Delta})$ prevents ${ }^{125} \mathrm{I}-\omega$-ctx degradation but not ${ }^{125} \mathrm{I}-\omega$-ctx internalization (inset), in undifferentiated SH-SY5Y cells. In these particular experiments, control half-lives $(\Theta)$ for degradation and internalization were, respectively, 17.3 and $14.4 \mathrm{hr}$. Halflives in the presence of leupeptin were, respectively, 115.0 and $15.5 \mathrm{hr}$. Similar results were obtained in five independent experiments.

known to affect the lysosomal degradation of other membrane proteins, also reduced ${ }^{125} \mathrm{I}$ - $\omega$-ctx degradation in a dose-dependent manner. In the presence of $20 \mu \mathrm{M}$ leupeptin, the degradation half-life was $38 \pm 4 \mathrm{hr}$ (mean $\pm \mathrm{SEM} ; n=4$ ) (Fig. 6).

While both chloroquine and leupeptin significantly reduced ${ }^{125} I-\omega$-ctx degradation, neither drug affected ${ }^{125} \mathrm{I}-\omega$-ctx internalization, which proceeded with rates similar to those of the controls (insets of Figs. 5 and 6, for chloroquine and leupeptin, respectively).

\section{VOCC turnover during neuroblastoma cell differentiation}

We have previously shown that both the number of ${ }^{125} I-\omega$-ctx binding sites and that of high-voltage-activated calcium currents increases during in vitro drug-induced neuroblastoma cell differentiation (Carbone et al., 1990). An increase in the number of surface channels can be achieved by an incrcase in protein synthesis and/or protein incorporation in the plasma membrane, or by a slowing down in protein internalization and degradation. To investigate whether channel turnover was modified during differentiation, we carried out experiments with ${ }^{125} l-\omega-$ ctx on differentiated cells.

In IMR 32 cells differentiated for $7 \mathrm{~d}$ in the presence of $1 \mathrm{~mm}$ dibutyryl-5-cyclic monophosphate and $2.5 \mu \mathrm{M}$ 5-bromodeoxyuridine, the half-life of ${ }^{125} \mathrm{I}-\omega$-ctx degradation was increased to $45 \pm 5 \mathrm{hr}$ (mean \pm SEM; $n=3$ ) (Fig. 7). No acute changes in the degradation rate were observed during the first $24 \mathrm{hr}$ of culture in the presence of the differentiating agents. The increase in half-life was gradual and developed over several days of exposure to the drugs $(16.8,37.6,45$, and $63 \mathrm{hr}$ after, respectively, 1, 3, 7, and 15 d). Similar results were obtained in SHSY5Y cells differentiated for several days in the presence of 10 $\mu \mathrm{M}$ retinoic acid (not shown).

As indicated by the acid wash experiments, the metabolic stability of VOCCs induced by cell differentiation was primarily due to a direct effect on the cellular mechanisms controlling VOCC internalization, which was significantly slowed down in differentiated cells (Fig. 7, inset), rather than to changes in the efficiency of the differentiated lysosomal apparatus. 


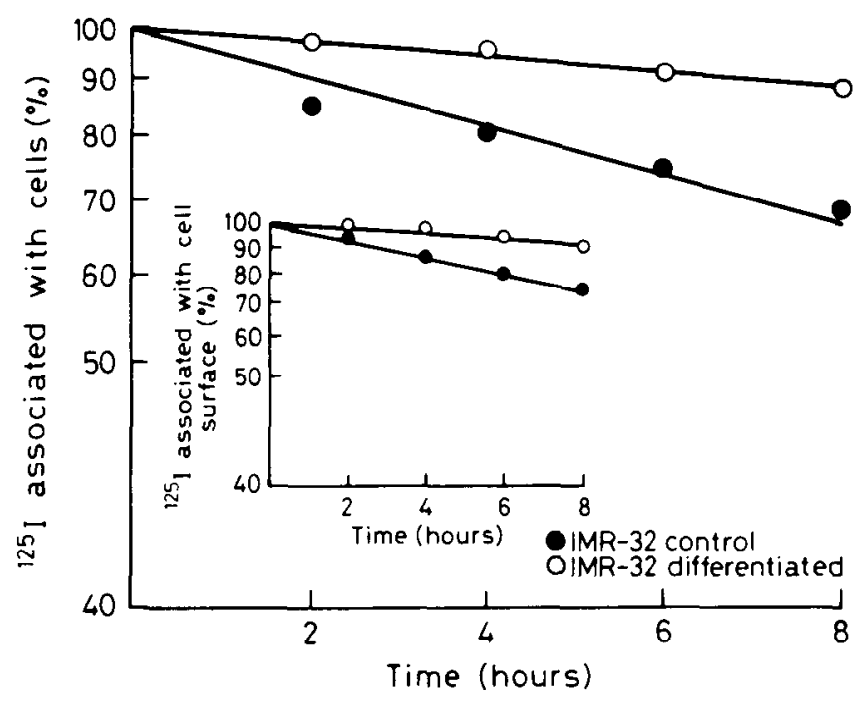

Figure 7. ${ }^{125} \mathrm{I}-\omega-\mathrm{Ctx}$ degradation and internalization in differentiated neuroblastoma cells. Both ${ }^{125} I-\omega$-ctx degradation and internalization (inset) rates were slowed in differentiated $(0)$ versus control $(\Theta)$ IMR32 cells. At time 0, ${ }^{125} \mathrm{I}-\omega$-ctx-specific binding was $1667 \mathrm{cpm} / \mathrm{dish}$ in control cells and $5282 \mathrm{cpm} /$ dish in differentiated cells. In these particular experiments, the degradation rates were 15.5 and $45.0 \mathrm{hr}$, and the internalization rates were 15.0 and $41.6 \mathrm{hr}$, for control and differentiated (for 7 d) cells, respectively.

\section{Effects of anti-channel antibodies on VOCC turnover}

In LEMS, autoantibodies are produced against presynaptic $\omega$-type VOCCs (Sher et al., 1989b), and these antibodies are known to affect the number and function of VOCCs in different in vivo and in vitro preparations (Vincent et al., 1989).

Two sets of anti-VOCC antibodies were used in order to study any effects they might have on VOCC turnover: one source of antibodies was the serum of LEMS patients; the other was the serum of mice immunized with intact neuroblastoma cells as described in Materials and Methods. Both antisera contained antibodies capable of precipitating ${ }^{125} \mathrm{I}-\omega$-ct $\mathrm{x}$-labeled VOCCs

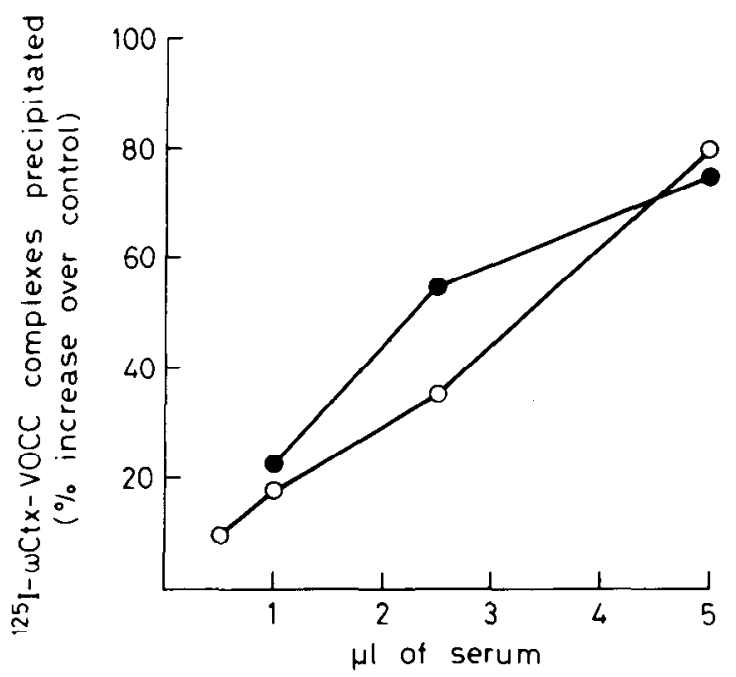

Figure 8. Immunoprecipitation of ${ }^{25} I-\omega$-ct $x$-labeled VOCCs from human neuroblastoma cells. Increasing concentrations of LEMS serum (•) or the serum of mice immunized with intact neuroblastoma cells $(O)$ werc reacted with ${ }^{125} I$ - $\omega$-ct $x$-labeled VOCCs solubilized from IMR32 cells. These sera had antibody titers (determined as described in Materials and Methods) of $110(0)$ and $80(\mathrm{O})$ pM against IMR32 VOCCs.

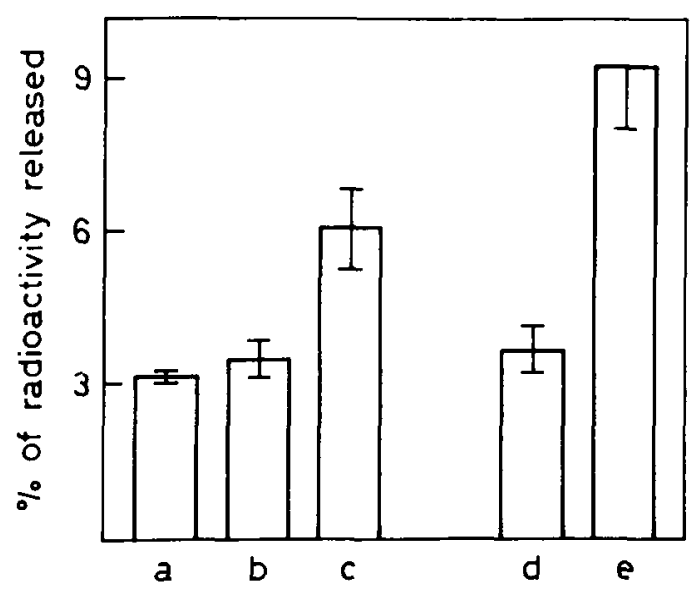

Figure 9. Degradation of ${ }^{125} \mathrm{I}-\omega-\mathrm{ctx}-\mathrm{VOCC}$ complexes in human neuroblastoma cells exposed to different anti-VOCC antisera. IMR 32 cells were prelabeled with ${ }^{125} \mathrm{I}-\omega$-ctx, and the radioactivity released in the medium after $1 \mathrm{hr}$ at $37^{\circ} \mathrm{C}$ was determined as described in Materials and Methods. Values represent the percentage of radioactivity released with respect to that specifically bound at time 0 (average $\pm \mathrm{SE} ; n=3$ ). IMR 32 cells were incubated in the presence of a $10 \%$ concentration of either fetal calf serum $(a)$, control human serum $(b)$, LEMS serum (c), control mouse serum $(d)$, or immunized mouse serum $(e)$.

extracted from IMR32 cells (Fig. 8). The anti- $\omega$-type VOCC antibody titer was 110 pM in LEMS serum and 80 pм in immunized mouse serum.

Both sera increased receptor turnover: in cells treated with LEMS serum, the half-life of ${ }^{125} \mathrm{I}-\omega$-ctx degradation was $11 \pm$ $1.2 \mathrm{hr}$ (mean $\pm \mathrm{SEM} ; n=3$ ) versus the $17 \pm 2 \mathrm{hr}$ (mean \pm $\mathrm{SEM} ; n=5$ ) of cells incubated in the presence of control human serum; in cells treated with mouse antiserum, the half-life of ${ }^{125} \mathrm{I}$ - $\omega$-ctx degradation was $14 \pm 0.8 \mathrm{hr}$ (mean $\pm \mathrm{SEM} ; n=3$ ) versus the $19 \pm 1.5 \mathrm{hr}$ (mean $\pm \mathrm{SEM} ; n=5$ ) of cells incubated in the presence of control mouse serum. The effect of the antiVOCC antibodies was rapid: after only $1 \mathrm{hr}$ of exposure to the antisera, a greater amount of radioactivity was released into the medium by prelabeled cells than by control cells (Fig. 9). The increased degradation of ${ }^{125} \mathrm{I}-\omega$-ctx induced by anti-VOCC antibodies was completely prevented by coincubation with $20 \mu \mathrm{M}$ chloroquine (not shown), thus indicating that both normal VOCC turnover (see above) and antibody-accelerated VOCC turnover occur in similar intracellular, acidic organelles.

\section{Discussion}

Synaptic activity is subject to both rapid regulatory events and more slowly developing adaptation processes. Onc of the molecular targets of these processes is the VOCC, a membrane protein that plays a crucial role in the control of cell excitability, neurotransmitter release, synaptogenesis, and synaptic pathology (Bean, 1989; Hess, 1990; Sher et al., 1991).

VOCC activity is acutely modulated by events such as phosphorylation/dephosphorylation or by interaction (via G-proteins) with other membrane receptors (Carbone and Swandulla, 1989). These rapid modulatory events occur, and revert, within a time scale of a few milliseconds. On a relatively longer time scale, a synapse might modulate its function by up- or downregulating the actual number of VOCCs expressed on the plasma membrane.

In a number of tissues, VOCC expression is subject to both 
homologous and heterologous regulation (Ferrante and Triggle, 1990). In particular, neuronal high-voltage-activated calcium channels are known to be upregulated in differentiating neurons (Streit and Lux, 1989; Carbone et al., 1990; Usowicz et al., 1990), to be concentrated in the growth cones (Lipscombe et al., 1988; Silver et al., 1990; Reber and Reuter, 1991), and to be clustered in the presynaptic active zones of mature synapses (Robitaille et al., 1990; Cohen et al., 1991; Torri Tarelli et al., 1991).

In order to obtain more information on the processes regulating VOCC expression, an in vitro model system combining the use of human neuroblastoma cells and $\omega$-ctx was adopted. Most of the experiments reported in this article were performed by following the fate of ${ }^{125} I-\omega$-ctx prebound to the cells because our results indicate that this is identical to the fate of the $\omega$-type VOCC:

(1) The degradation half-life of specifically bound toxin is very similar to the half-life of toxin binding site removal from the plasma membrane in cycloheximide-treated cells.

(2) The loss of toxin binding sites from the plasma membrane is parallel to the loss of functional $\mathrm{Ca}^{2+}$ channels measured with the fura-2 technique in cycloheximide-treated cells.

(3) $\mathrm{A} \mathrm{pH}$ as low as $\mathrm{pH} 3$ is necessary to detach a significant amount of specifically bound toxin from the cell surface, making it highly unlikely that the toxin dissociates from the channel in endosomes or other intracellular organelles whose internal $\mathrm{pH}$ is between 4 and 6 .

(4) The appearance of radioactivity in the medium and the corresponding loss of cell-associated radioactivity are clearly a consequence of the metabolic processes of the cell itself and not of the dissociation of prebound ${ }^{125} \mathrm{I}-\omega$-ctx or nonspecific toxin degradation because the radioactivity in the medium was found to be associated with toxin degradation products and, furthermore, its catabolism was blocked by reducing the temperature, by increasing the $\mathrm{pH}$ of intracellular organelles, and by blocking lysosomal proteases.

(5) Antibodies against $\omega$-type VOCCs (from both LEMS patients and from immunized mice) increased ${ }^{129} 1-\omega$-ctx catabolism, and this accelerated catabolism was slowed by chloroquine in the same way as in cclls not treated with antibody.

The metabolism of $\omega$-type VOCCs in neurons is similar in many respects to that of muscle nicotinic receptors (Fambrough, 1979). The calculated VOCC internalization and degradation half-lives are similar to those obtained in vitro for other membrane ion channels, such as the nicotinic receptor (Devreotes and Fambrough, 1975) and the voltage-operated sodium channel (Sherman and Catterall, 1984; Bar-Sagi and Prives, 1985; Dargent and Couraud, 1990). This suggests that there is a general feature that underlies the turnover of this particular class of membrane proteins.

The mechanisms behind the increased expression of plasma membrane $\omega$-type VOCCs in differentiated neurons are particularly interesting, because this could be achieved by different mechanisms alternatively influencing protein synthesis or degradation. Both possibilities have been described in the case of muscle nicotinic reccptors: the increased expression of extrajunctional nicotinic receptors in developing myotubes has been shown to be due to increased synthesis, with no changes in the degradation rate (Devreotes and Fambrough, 1975); and the increased expression of junctional nicotinic $\mathrm{ACh}$ receptors induced by cAMP and CAMP analogs in denervated muscles has been found to be caused by a decrease in the receptor degradation rate (Shyng et al., 1991).

By using different and unrelated differentiating drugs in two different human neuroblastoma cell lines, we have found that the morphological differentiation of the cells was accompanied by a parallel decrease in VOCC catabolism that was due to reduced channel internalization. The molecular mechanisms of this "stabilization" are still unknown, although posttranslational channel modifications (e.g., phosphorylations), changes in channel subunit composition, and the induction of specific interactions with the cytoskeleton are all possibilities worth investigating.

VOCC turnover was slowed down during differentiation, but it could also be pathologically accelerated. LEMS autoantibodies and mouse anti- $\omega$-type VOCC antibodies induced an increased internalization and degradation of $\omega$-type VOCCs. At the cellular level, these results confirm previous suggestions that LEMS autoantibodies may act by cross-linking VOCCs (Nagel et al., 1988) and thus inducing VOCC downregulation (Sher et al., 1989b; Leys et al., 1991).

The antibody effects described here were more rapid than might be expected from the results of previous studies concerning the effects of antibodies on ${ }^{45} \mathrm{Ca}^{2+}$ influx (Roberts et al., 1985; Dc Aizpurua ct al., 1988) or VOCC downregulation (Sher et al., 1989b; Leys et al., 1991), probably because the present experiments studied the selective effects of the antibodies on prelabeled channels, without the interference of newly incorporated channels.

LEMS antibody-induced VOCC downregulation has already been shown to be specific in comparison with other membrane molecules (Sher et al., 1989b), a highly selective, antibody-induced downregulation of membrane receptors and ion channels being a common feature of a number of human autoimmune disorders (Clementi and Sher, 1987).

\section{References}

Ahlidanian MK, Westenbroek RE, Catterall WA (1990) Subunit structure and localization of dihydropyridine-sensitive calcium channels in mammalian brain, spinal cord and retina. Neuron 3:819-832.

Bar-Sagi D, Prives J (1985) Negative modulation of sodium channels in cultured chick muscle cells by the channel activator batrachotoxin. J Biol Chem 260:4740-4744.

Bean BP (1989) Classes of calcium channels in vertebrate cells. Annu Rev Physiol 51:367-384.

Carbone E, Swandulla D (1989) Neuronal calcium channels: kinetics, blockade and modulation. Prog Biophys Mol Biol 54:31-58.

Carbone E, Sher E, Clementi F (1990) Ca currents in human neuroblastoma IMR32 cells: kinetics, permeability and pharmacology. Pfluegers Arch 416:170-179.

Clementi F, Sher E (1987) Antibody induced down regulation of membrane receptors in human diseases. In: Molecular mechanisms of desensitization to signal molecules (Konijn TM, Van Haastert PJM, Van der Stane H, Van der Vel H, Houslay MD, eds), pp 301-317. Berlin: Springer.

Cohen MW, Jones OT, Angelides KJ (1991) Distribution of $\mathrm{Ca}^{2+}$ channels on frog motor nerve terminals revealed by fluorescent $\omega$-conotoxin. J Neurosci 11:1032-1039.

Dargent B, Couraud F (1990) Down-regulation of voltage-dependent sodium channels initiated by sodium influx in developing neurons. Proc Natl Acad Sci USA 87:5907-5911.

De Aizpurua HJ, Lambert EH, Griesmann GE, Olivera BM, Lennon VA (1988) Antagonism of voltage-gated calcium channels in small cell carcinomas of patients with and without Lambert-Eaton myasthenic syndrome by autoantibodies, $\omega$-conotoxin and adenosine. Cancer Res 48:4719-4724.

Devreotes PN, Fambrough DM (1975) Acetylcholine receptor turn- 
over in membranes of developing muscle fibers. J Cell Biol 65:335358.

Di Virgilio F, Milani D, Leon A, Meldolesi J, Pozzan T (1987) Voltagedependent activation and inactivation of calcium channels in PC12: correlation with neurotransmitter release. J Biol Chem 262:91899195.

Fambrough DM (1979) Control of acetylcholine receptors in skeletal muscle. Physiol Rev 59:165-227.

Ferrante J, Triggle DJ (1990) Homologous and heterologous regulation of voltage-dependent calcium channels. Biochem Pharmacol 39:12671270 .

Hess P (1990) Calcium channels in vertebrate cells. Annu Rev Neurosci 13:337-356.

Higgins LS, Berg DK (1988) Cyclic AMP-dependent mechanism regulates acetylcholine receptor function on bovine adrenal chromaffin cells and discriminates between new and old receptors. J Cell Biol 107:1157-1165.

Leys K, Lang B, Johnston I, Newsom-Davis J (1991) Calcium channel autoantibodies in the Lambert-Eaton myasthenic syndrome. Ann Neurol 29:307-314.

Lipscombe D, Madison DV, Poenie M, Reuter H, Tsien RY, Tsien RW (1988) Spatial distribution of calcium channels and cytosolic calcium transients in growth cones and cell bodies of sympathetic neurons. Proc Natl Acad Sci USA 85:2398-2402.

Miller RJ (1987) Multiple calcium channels and neuronal function. Science 235:46-52

Nagel A, Engel AG, Lang B, Newsom-Davis J, Fukuoka T (1988) Lambert-Eaton myasthenic syndrome IgG depletes presynaptic mcmbrane active zone particles by antigenic modulation. Ann Neurol 24:552-558.

Olivera BM, Rivier J, Clark C, Ramilo CA, Corpuz GP, Abogafie FC, Mena EE, Woodward SR, Hillyard DR, Cruz LJ (1990) Diversity of Conus neuropeptides. Science 249:257-263.

Pahlman S, Ruusala A, Abrahamsson L, Mattsoon MEK, Esscher T (1984) Retinoic acid-induced differentiation of cultured neuroblastoma cells: a comparison with phorbolester-induced differentiation. Cell Differ 14:135-144.

Paul S, Volle DJ, Beach CM, Johnson DR, Powell MJ, Massey RJ (1989) Catalytic hydrolysis of vasoactive intestinal peptide by human autoantibody. Science 244:1158-1162.

Reber BFX, Reuter H (1991) Dependence of cytosolic calcium in differentiating rat pheochromocytoma cells on calcium channels and intracellular stores. J Physiol (Lond) 435:145-162.

Roberts A, Perera S, Lang B, Vincent A, Newsom-Davis J (1985) Paraneoplastic myasthenic syndrome IgG inhibit ${ }^{45} \mathrm{Ca}^{2+}$ flux in a human small carcinoma line. Nature 317:737-739.

Robitaille R, Adler EM, Charlton MP (1990) Strategic location of calcium channels at transmitter release sites of frog neuromuscular synapses. Neuron 5:773-779.
Sher E, Clementi F (1991) $\omega$-Conotoxin-sensitive voltage-operated calcium channels in vertebrate cells. Neuroscience 42:301-307.

Sher E, Pandiella A, Clementi F (1988) $\omega$-Conotoxin binding and effects on calcium channel function in human neuroblastoma and rat pheochromocytoma cell lines. FEBS Lett 235:178-182.

Sher E, Denis-Donini S, Zanini A, Bisiani C, Clementi F (1989a) Human neuroblastoma cells acquire regulated secretory properties and different sensitivity to $\mathrm{Ca}^{2+}$ and $\alpha$-latrotoxin after exposure to differentiating agents. J Cell Biol 108:2291-2300.

Sher E, Gotti C, Canal N, Scoppetta C, Piccolo G, Evoli A, Clementi F (1989b) Specificity of calcium channel autoantibodies in LambertEaton myasthenic syndrome. Lancet II:640-643.

Sher E, Biancardi E, Passafaro M, Clementi F (1991) Physiopathology of neuronal voltage-operated calcium channels. FASEB J 5:26772683.

Sherman SJ, Catterall WA (1984) Electrical activity and cytosolic calcium regulate levels of tetrodotoxin-sensitive sodium channels in cultured rat muscle cells. Proc Natl Acad Sci USA 81:262-266.

Shyng S-L, Xu R, Salpeter MM (1991) Cyclic AMP stabilizes the degradation of original junctional acetylcholine receptors in denervated muscle. Neuron 6:469-475.

Silver RA, Lamb AG, Bolsover SR (1990) Calcium hotspots caused by $L$-channel clustering promote morphological changes in neuronal growth cones. Nature 343:751-755.

Streit J, Lux HD (1989) Distribution of calcium currents in sprouting PC.12 cells. J Neurosci 9:4190-4199.

Swandulla D, Carbone E, Lux HD (1991) Do calcium channel classifications account for neuronal calcium channel diversity? Trends Neurosci 14:46-51.

Thompson JM, London ED, Johnson JR (1982) Ultrastructural, functional and biochemical characteristics of mouse and human neuroblastoma cell lines. Neuroscience 7:1807-1815.

Torri Tarelli F, Passafaro M, Clementi F, Sher E (1991) Presynaptic localization of $\omega$-conotoxin-sensitive calcium channels at the frog neuromuscular junction. Brain Res 547:331-334.

Usowicz MM, Porzig H, Becker C, Reuter H (1990) Differential expression by nerve growth factor of two types of $\mathrm{Ca}^{2+}$ channels in rat phaeochromocytoma cell lines. J Physiol (Lond) 426:95-116.

Van Deurs B, Peterson OW, Sjur O, Kirsten S (1989) The ways of endocytosis. Int Rev Cytol 117:131-177.

Vincent A, Lang B, Newsom-Davis J (1989) Autoimmunity to the voltage-gated calcium channel underlies the Lambert-Eaton myasthenic syndrome, a paraneoplastic disorder. Trends Neurosci 12:496502.

Vuk-Pavlovic S, Kovach JS (1989) Recycling of tumor necrosis factor$\alpha$ receptor in MCF-7 cells. FASEB J 3:2633-2640.

Westenbroek RE, Ahlijanian MK, Catterall WA (1990) Clustering of L-type $\mathrm{Ca}^{2+}$ channels at the base of major dendrites in hippocampal pyramidal neurons. Nature 347:281-284. 